\title{
Sensitivity analysis of water consumption in an office building
}

\author{
Tomas Suchacek ${ }^{1, *}$, Ladislav Tuhovcak ${ }^{1}$, and Jan Rucka $^{1}$ \\ ${ }^{1}$ Brno University of Technology, Faculty of Civil Engineering, Institute of Municipal Water \\ Management, Zizkova 17, 60200 Brno, Czech republic, France
}

\begin{abstract}
This article deals with sensitivity analysis of real water consumption in an office building. During a long-term real study, reducing of pressure in its water connection was simulated. A sensitivity analysis of uneven water demand was conducted during working time at various provided pressures and at various time step duration. Correlations between maximal coefficients of water demand variation during working time and provided pressure were suggested. The influence of provided pressure in the water connection on mean coefficients of water demand variation was pointed out, altogether for working hours of all days and separately for days with identical working hours.
\end{abstract}

\section{Introduction}

In the Czech Republic, there is currently no legislative regulation defining in a mandatory manner a detailed procedure of calculating water demand related to a public main supplying water to a service area of a town or village type. Traditionally, a combination of empiric experience and partial legislative regulations is employed to determine the volume of water needed for the specific types of buildings or drinking water consumers in units of volume of water per calendar year. Nevertheless, when designing a water supply network, it is necessary to meet the legislative requirements which, in fact, define the hydraulic parameters of the water network. Given the defined design flow-rate it is necessary to ensure the required range of operating pressures in those parts of the water network where consumers are connected. If the water supply is also used for fire-fighting purposes, the required pressure and flow-rate must be ensured for fire water supply. For comprehensive water supply systems, the design or redesign is often based on mathematical modelling and simulation methods [1], where the hydraulic calculations use as inputs also the daily and hourly variation coefficients (demand coefficients). The value of these coefficients is not set in legislation of the Czech Republic, not even with the implementation of legislative norms on the EU level (e.g. CSN EN 805). It is important to define (design flow) for separate sections or whole networks. In the Czech Republic, the design of water distribution network is calculated with maximal water consumption per hour. The method of calculating maximal water consumption per hour is anchored in legislation, in which the coefficient of maximal variation per hour figures. The value of this coefficient is thus, extremely

*Corresponding author: suchacek.t@ffce.vutbr.cz 
important for the design of hydraulic capacity of water distribution network. According to legislation, its value can only vary with the number of supplied residents, therefore the focus is set on the maximal coefficients of water demand variation during simulated changes of pressure conditions in the network.

Absence of the coefficients of water demand variation prove to be problematic mainly while designing new networks, when recommended and established values of hourly coefficients of variation are used. But even if the demand coefficients are specified (e.g. in historical and obsolete legislative), the fact that the values of these coefficients might be modulated by various pressure conditions is not taken into consideration. Therefore, they are considered constant, in spite the fact that pressure influences the water consumption. For example, according to [2], water demand is divided for the purposes of simulating water demands changes in relation to pressure changes into "inside the house" and "outside the house". Both these parts of demand have a different coefficient expressing the dependency on pressure while the "outside the house" demand is much more influencable by pressure. Subsequently, the average coefficient is calculated expressing the dependency of water demand on pressure for the entire building. This coefficient is then implemented in the FAVAD equation [3]. The coefficient value for the "inside the house" demand was set for example in [4] for the student campus in Johannesburg at 0.2. According to [5], the dependency of demand on pressure was also proved for pressure toiler flushers in Great Britain and the coefficient value was set at 0.07 and 0.025 . For example, for the "outside the house" consumption, the value of the coefficient according to $[6,7]$ was set to approximately 0.5 , with domestic irrigation systems.

Another way of consumption division for the prediction is, for example, the division according to [8] into a part dependent on pressure and a part dependent on volume. Subsequently, depending on the ratio of both parts of the consumption, dependency is determined for the change of consumption with the change of pressure. According to [9] it is possible to describe a pressure dependence using pressure threshold values. These threshold values and the reference pressure are minimal, and it is assumed that when the pressure drops below the minimal threshold value, the consumption is zero, on the other hand, when the reference value is exceeded, the consumption is constant and with increasing pressure.

The pressure conditions in the water distribution network or the pressure is the most important operating factor that affects water consumption, but on the other hand, the water consumption is influenced by other factors of different kinds. For example, according to [10], the consumption is characterized based on the building characterization. Another group is climatological factors, especially the influence of temperature and precipitation as well as partly humidity, for example according to [11, 12].

For these reasons, attention is also paid to coefficients of water demand variation during working hours in response to simulated modulation of pressure conditions.

The aim of this study was to study change in variation of water demand with the variation of pressure in the water connection, to which the building is connected by installing a pressure reducing valve on this water connection and to observe and evaluate water consumption, or its variation, respectively.

\section{Methodology}

The primary requirement for calculating the influence of pressure conditions on the water consumption, and especially its variation is the modification of the pressure conditions. In this case, the modification has not been applied in the whole water distribution network, but by installing a pressure reducing valve onto the water connection. The valve simulated the regulation of pressure conditions in the whole network. The output value of the pressure 
reducing valve was randomly selected by random number generator. Restricting conditions on the minimal output pressure were applied, so that no legislative regulations concerning minimal hydrodynamic pressure would be violated, ensuring usual working flow of the respective building.

For execution of measuring campaign, so called measuring cycles were defined. Settings of the pressure reducing valve would remain constant for the duration of a cycle. Duration of a single cycle was defined to approximately 14 days, which usually represents 10 working days in a single cycle.

In order to be able to register potential decreases in hydrodynamic pressure under the actual value of output pressure and other eventual anomalies in the network, a pressure record interval of 15 second was chosen. For recording volume of water per time unit, a water meter with pulse generator, whose value was set to 1 litre so that water categorization into shorter time intervals would be possible. The value of the water meter was recorded every 15 seconds.

As the campaign lasted 1 year, it was necessary to take into account or exclude longterm impacts and trends in water consumption. In the Czech Republic, there has been a downward trend in water consumption since 1989 but in the last 3 years consumption can be considered more or less stable [13]. The effect of long-term trends could thus be excluded. For exclusion of extremely non-standard or anomalous measurements during working days, number of workers in the building during working hours was monitored. From the statistical data set, days during which the number of workers dropped under $10 \%$ of the total amount were excluded. Similar days could discredit the results of the study.

Data results were assessed using two methods. Firstly, maximal data set was used in the statistical file by including all days without taking the length of working time into consideration. In order to be able to make use of data from all days, only shared working hours among all days were taken into account. The second method of result assessment was done separately for days with identical working hours and coefficient was determined for the whole duration of the working time. Evaluation and determination of coefficients were done by the following equations:

Flow in the time step $i$, in day $j$ is determined as average flow in day $j$ multiplied by coefficient in the time step $i$ during day $k$.

$$
Q_{i j}=Q_{M E A N_{j}} c_{i j}
$$

where $Q_{i j}\left[1\right.$. hour $\left.{ }^{-1}\right]$ represents flow, $Q_{M E A N j}\left[1\right.$. hour $\left.^{-1}\right]$ is mean flow, $c_{i j}[-]$ is coefficient of water demand variation, $i$ is the label of the time step and $j$ is the label of the day.

Coefficient of variation after modification is determined as follows:

$$
c_{i j}=\frac{Q_{i j}}{Q_{M E A N_{j}}}
$$

Mean coefficient for each time step in pressure category $k$ is determined as the sum of all coefficient values in time step $i$, in the pressure category $k$.

$$
c_{\text {MEAN } i k}=\frac{\sum_{j=1}^{m} c_{i j k}}{m_{k}}
$$


where $c_{\text {MEANik }}$ [-] is mean coefficient, $k$ is pressure category (as seen below), $m_{k}[-]$ is the total number of days in category $k$.

$$
c_{M A X_{k}}=\max \left\{c_{i j k}\right\}
$$

where $c_{\text {MAXk }}[-]$ is the maximal coefficient in $k$ category.

Categorization of measured mean pressures was done with regard to balancing values in the respective categories, as well as with regard to size of intervals of the categories. Too delicate division would mean relatively small, or even no representation in some pressure categories.

$$
\varepsilon_{k}=\sum_{i=1}^{n}\left(c_{i k}-1\right)^{2}
$$

where $\varepsilon_{k}[-]$ represents square deviation of coefficients from optimal value in each time step.

\section{Case study}

Case study was conducted on an office building, which is a relatively common type of building in a consumption area up to the size of a regional city. This building has three above-ground floors and the maximal number of workers is 35 . The workers are practically evenly divided into each of the above-ground floors, in which identical equipment is provided. For anomaly determining in measured statistical data set, the number of persons in the building was monitored during the working days. The evaluation of correlation was done only for the working days. The duration of the measuring campaign in the case study was one calendar year. According to survey, it was found out that in the sense of [2], all measured water was consumed "inside the house".

There are three working time shifts in the studied building: 7:00 to 17:00 on Mondays and Wednesdays, 7:00 to 15:00 on Tuesdays and Thursdays and 7:00 to 13:00 on Fridays. For the first method of evaluation, shared working hours (from 7:00 - 13:00) from all days were taken into account. For the second method, working hours of each respective day were assessed separately.

The following values of water consumption were characteristic for the studied building.

Table 1. Characteristic values of water consumption during working hours

\begin{tabular}{|c|c|c|c|}
\hline $\begin{array}{c}\text { Water consumption in } \\
\text { working hours }\end{array}$ & $\begin{array}{c}\text { Minimal } \\
\text { consumption }\end{array}$ & $\begin{array}{c}\text { Mean } \\
\text { consumption }\end{array}$ & $\begin{array}{c}\text { Maximal } \\
\text { consumption }\end{array}$ \\
\hline Day (working hours) & (litres) & (litres) & (litres) \\
\hline Monday + Wednesday (7-17) & 608 & 892 & 1307 \\
\hline Tuesday + Thursday (7-15) & 481 & 710 & 1252 \\
\hline Friday (7-13) & 307 & 623 & 927 \\
\hline All days (7-13) & 333 & 545 & 982 \\
\hline
\end{tabular}


For this case study, the intervals of pressure conditions categories and values in individual categories is shown in Figure 1. The value of pressure for one day was determined as arithmetic mean during the working hours of the respected day.

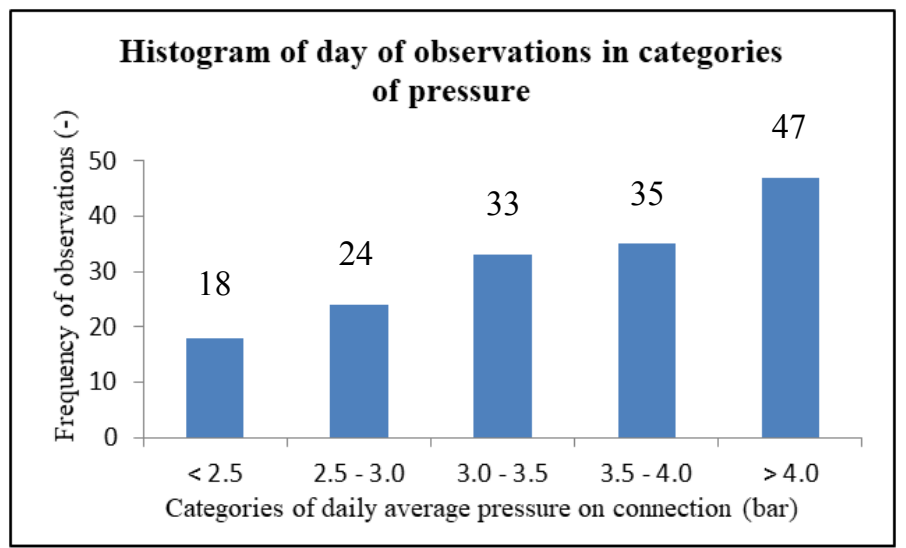

Fig. 1. Histogram of measured values in pressure categories

As a part of this case study, a sensitivity analysis of duration of a time step for maximal and mean coefficient of water demand variation was conducted. Time step durations fluctuate about the most frequent duration of a time, which is 60 minutes, or 1 hour. In this case, correlations for the time step with duration of 10, 20, 30, 40, 60, 90 and 120 minutes were studied.

\section{Results}

As stated above, in the first study method, shared working hours, from 7:00 - 13:00, of all days were taken into account (with the exception of anomalous values). From the coefficients for individual time steps in each pressure category, mean coefficients for the respective time steps were determined. Using equation (5), square deviation from optimal value was determined. For results presentation, pressure categories 2.5-3.0 bar and 3.5-4.0 bar were selected, and time step durations of 10,60 and 120 minutes were used. Coefficients of water demand variation were as follows: 


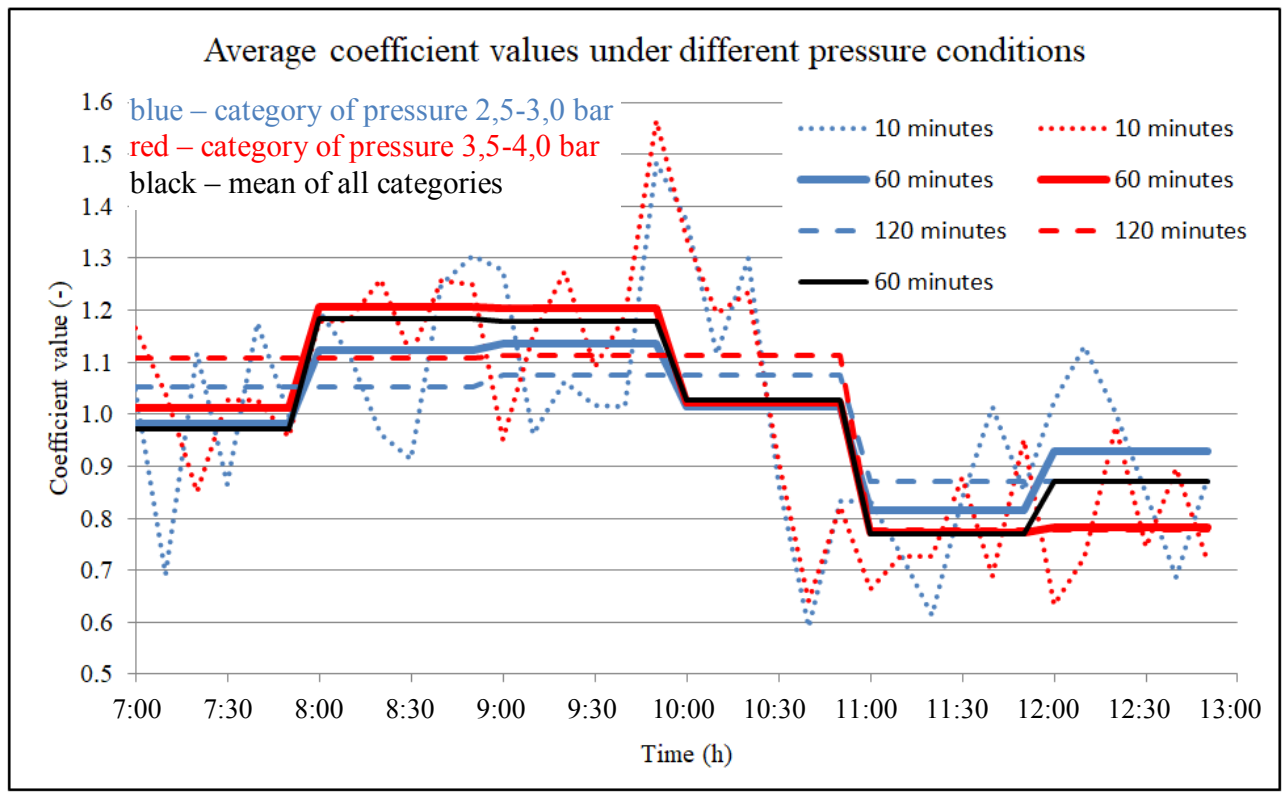

Fig. 2. Mean coefficients in relation in correlation to pressure - all days

Calculated square deviation values for selected categories are presented in the following table:

Table 2. Square deviation values - all days

\begin{tabular}{|c|c|c|c|c|c|c|c|}
\hline Time step & $\mathbf{1 0}$ & $\mathbf{2 0}$ & $\mathbf{3 0}$ & $\mathbf{4 0}$ & $\mathbf{6 0}$ & $\mathbf{9 0}$ & $\mathbf{1 2 0}$ \\
\hline $\begin{array}{c}\text { Category of } \\
\text { pressure (bar) }\end{array}$ & $\mathbf{( m i n )}$ & $\mathbf{( m i n )}$ & $\mathbf{( m i n )}$ & $\mathbf{( m i n )}$ & $\mathbf{( m i n )}$ & $\mathbf{( m i n )}$ & $\mathbf{( m i n )}$ \\
\hline $2.5-3.0$ & 1.599 & 1.086 & 0.975 & 0.762 & 0.444 & 0.306 & 0.300 \\
\hline $3.5-4.0$ & 1.908 & 1.589 & 1.487 & 1.343 & 1.103 & 0.845 & 0.890 \\
\hline
\end{tabular}

In the first study method, maximal coefficients of water demand variation were determined by equation (4). Evaluation of correlation between maximal coefficients and pressure are presented in Figure 3.

For the time step duration of 60 minutes, the maximal coefficient value in the lowest hydrodynamic pressure category was determined as 1.73 , while the maximal coefficient value in the highest hydrodynamic pressure category was determined as 2.14 , which signifies fluctuation of $26 \%$. 


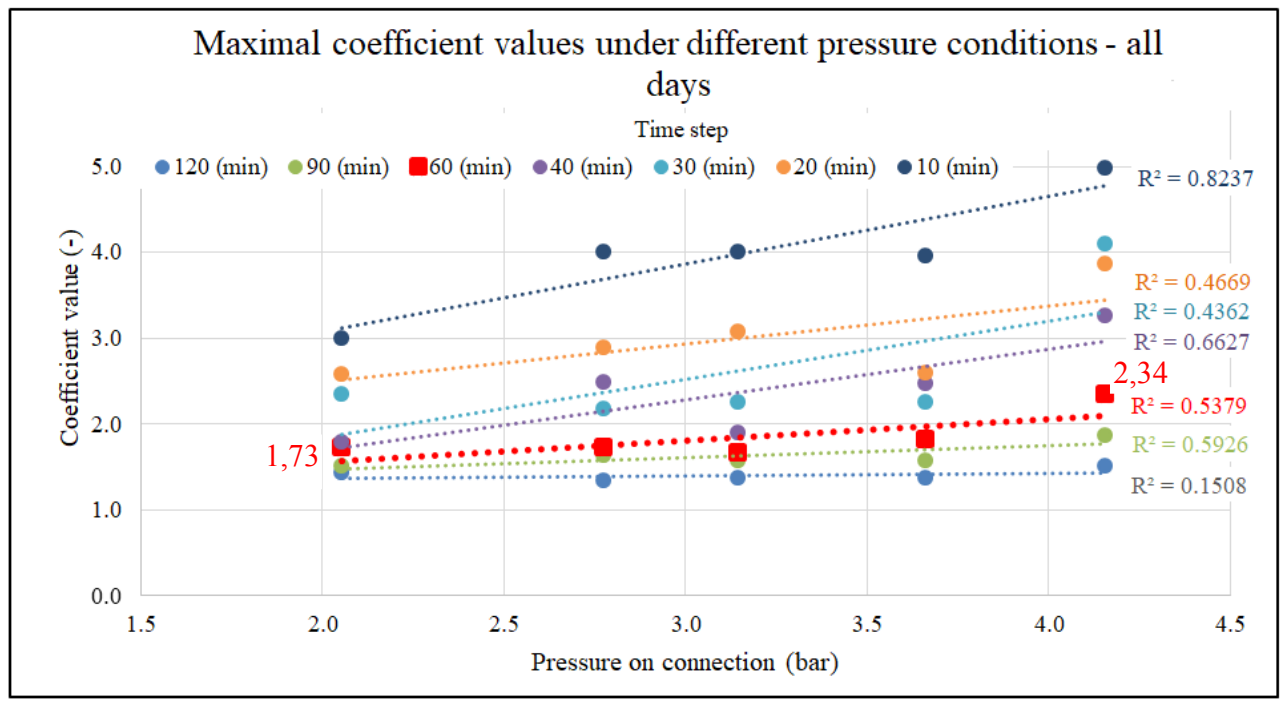

Fig. 3. Maximal coefficients of water demand variation - all days

In the second study method, only days with identical working hours were considered out of the statistical data set of evaluated data, and the whole of working time was taken into account. For results presentation of the second assessment method, pressure categories of 2.5-3.0 bar and 3.5-4.0 bar were selected, and time step durations of 30, 60 and 90 minutes were used. The days selected for results presentation were Tuesday and Thursday (working time from 7:00 to 15:00). Coefficients of water demand variation were as follows:

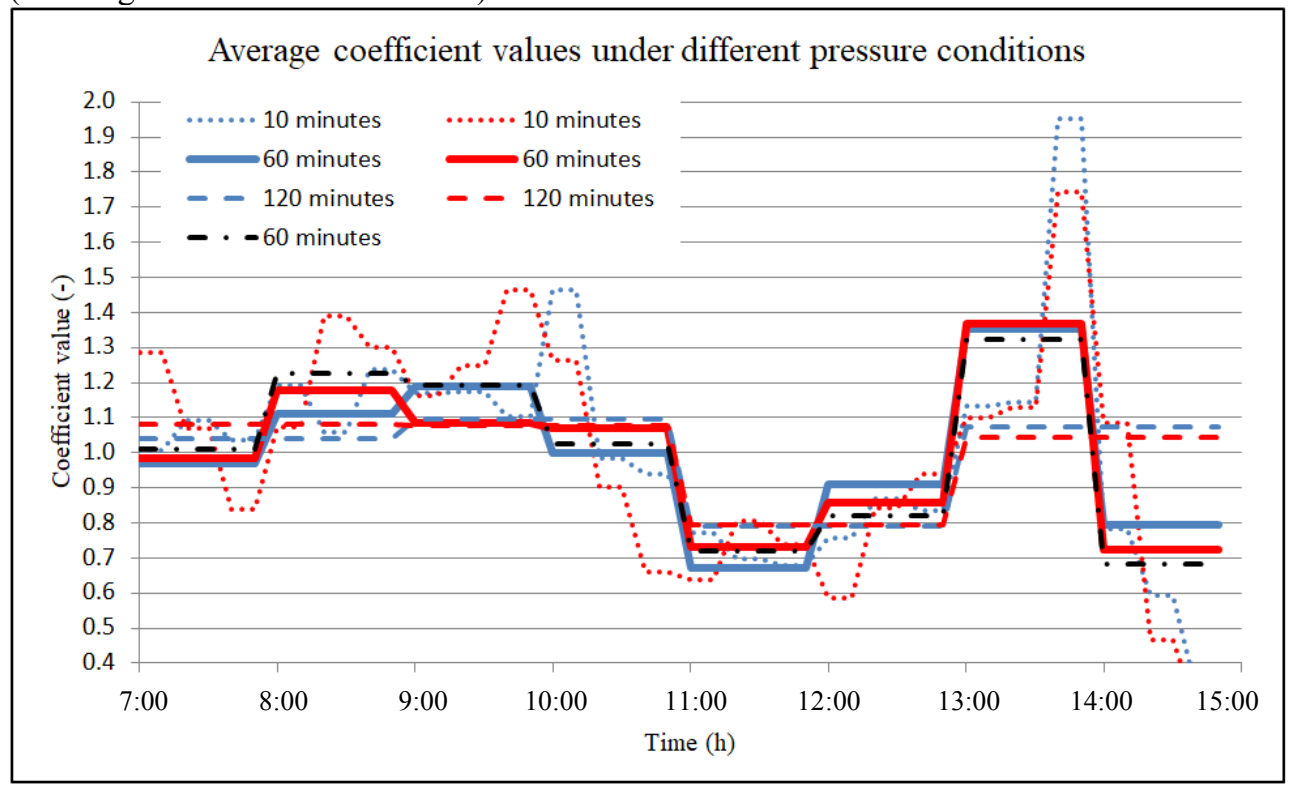

Fig. 4. Mean coefficients in relation to pressure - Tuesday and Thursday

In the second assessment method, square deviations were not determined, since the statistical data set was significantly smaller than in the first method. However, maximal coefficients of water demand variation were determined, as seen in Figure 5. 


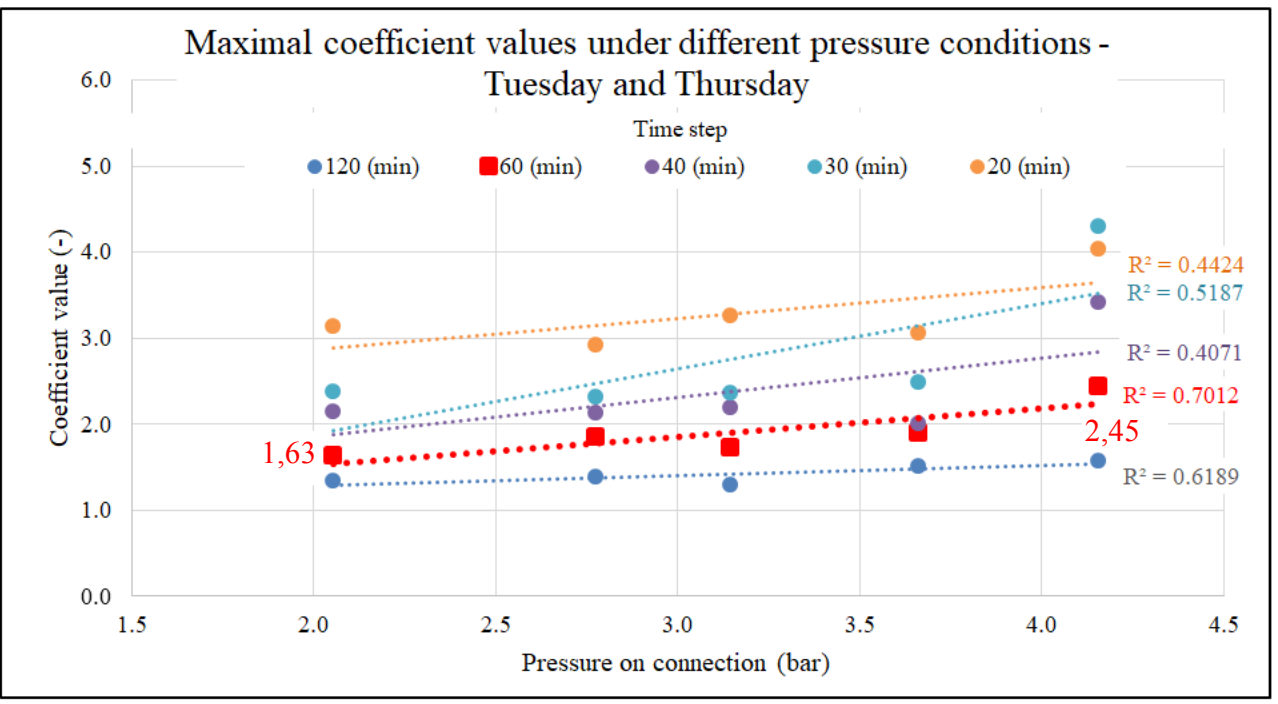

Fig. 5. Maximal coefficients of water demand variation - Tuesday and Thursday

For the time step duration of 60 minutes, the maximal coefficient value in the lowest hydrodynamic pressure category was determined as 1.63 , while the maximal coefficient value in the highest hydrodynamic category was determined as 2.45 , which signifies fluctuation of $33 \%$.

\section{Discussion}

Nowadays, there are only few real studies that deal with the issue of uneven water distribution depending on the different pressure conditions. Also, the value of the maximum hourly coefficient is important, according to which distribution networks are proposed, for example, in the Czech Republic. Operational control of pressure conditions could be used as a tool to reduce the uneven distribution from the distribution network, but for the proper management a large number of real studies are needed to be carried out to capture the characteristics of different types of customers. The problem of conducting realistic studies is the need to change the pressure conditions, which can cause customer complaints at the lower and upper limits of the allowed pressure range in the distribution network even if the legal requirements are met.

Although the area of water consumption dependence on pressure ratios and, respectively, unevenness of this consumption is still not fully documented, there are many possibilities to carry out more real measurements in the near future. This option is based on the use of SMART metering, which will allow to collect water consumption information at short time intervals for different types of customers. From these data, it would be possible in the future to be able to compile complex dependences of the unevenness of the distribution for the whole distribution network, not just for individual types of customers.

\section{Conclusions}

As seen from the aforementioned, in both methods of assessment, a certain correlation between pressure and variation of water demand is observed. This is apparent with both maximal and mean variation coefficient values, as presented in Table 2, where it is clear that fluctuation is higher with higher simulated water pressure in the water distribution 
network. It is then apparent that when predicting response to water distribution network behaviour, the pressure management of the networks should also take fluctuations of water demand time characteristics into account. Reducing pressure in the network has impact on water losses respectively on their reduction and the consumed volume of water. The influence of pressure in the water connection on the variation of water consumption in the studied building was proved. In this case, it was confirmed that the difference between maximal measured coefficients of water demand variation for lowest and highest categories was $26 \%$ at the most common time step duration of 60 minutes for all working days, and $33 \%$ for Tuesdays and Thursdays. Findings from this real study for a single water connection should then be implemented, with certain restrictions, for entire water distribution networks, or consumption areas, respectively. It is thus apparent that a detailed analysis of water consumption in time in correlation to pressure conditions, and their following prediction, is a contemporary issue.

\section{Acknowledgements}

This research work is funded by the Brno University of Technology in the frame of the research projects titled FAST-J-17-4348 Influence of boundary conditions on optimization of pressure conditions in water supply systems and FAST-S-17-4643 Modelling of selected indicators and processes in the supply of drinking water to users. Thanks to the town of Valasske Klobouky.

\section{References}

1. J. Kovář, J. Ručka and O. Andrš, Proceedings of the $16^{\text {th }}$ International Conference on Mechatronics - Mechatronika 2014, 697-700 (Brno, 2014)

2. A. Lambert and M. Fantozzi, Water Loss 2010 (Sao Paolo, 2010)

3. J. May, W. Wat. Env. Eng. (1994)

4. L. Bartlett, B.Eng Final Year Project Report. Pressure dependent demands in Student Town Phase 3 (Dept. of Civil and urban Engineering, Rand Afrikaans University, Johannesburg, 2004)

5. J. Thorton and A. Lambert, Leakage 2005 (Halifax, 2005)

6. Bamezai, Lessick, Water Conservation through System Pressure Optimization in Irvine Ranch Water District (2003)

7. R. Cullen, Pressure vs Consumption Relationships in Domestic Irrigation Systems (2004)

8. V. Kanakoudis and K. Gonelas, Proc. Eng., 89, 318-325 (2014)

9. J. M. Wagner, U. Shamir and D. H. Marks, J. Wat. Res. Plan. and Man., 114, 276-294 (1988)

10. W. A. Hussien, F. A. Memon and D. A. Savic, Wat. Res. Man., 30(9), 2931-2955 (2016)

11. M. Xenochristou, Z. Kapelan, C. Hutton and J. Hofman, CCWI 2017 - Computing and Control for Water Industry (Sheffield, 2017)

12. T. Suchacek, L. Tuhovcak, J. Rucka, CCWI 2017 - Computing and Control for Water Industry (Sheffield, 2017)

13. J. Duda, O. Lípa, T. Petr and V. Skácel, VODOVODY KANALIZACE ČR 2015, in Czech (Department of Agriculture, Prague, 2016) 